\title{
Effect of process parameters on surface texture generated by laser polishing of additively manufactured Ti-6Al-4V
}

\author{
J. S. Solheid ${ }^{\mathrm{a} *}$, A. Elkaseer ${ }^{\mathrm{b}, \mathrm{c}}$, T. Wunsch ${ }^{\mathrm{d}}$, A. P. Charles ${ }^{\mathrm{b}}$, H. J. Seifert ${ }^{\mathrm{a}}$, W. Pfleging ${ }^{\mathrm{a}, \mathrm{e}}$ \\ ${ }^{a}$ Institute for Applied Materials-Applied Materials Physics, Karlsruhe Institute of Technology, \\ P.O. Box 3640, 76021 Karlsruhe, Germany; \\ ${ }^{\mathrm{b}}$ Institute for Automation and Applied Informatics, Karlsruhe Institute of Technology, \\ P.O. Box 3640, 76021 Karlsruhe, Germany; \\ ${ }^{c}$ Faculty of Engineering, Port Said University, Port Fuad 24256, Egypt; \\ ${ }^{\mathrm{d}}$ Institute for Micro Process Engineering, Karlsruhe Institute of Technology, \\ P.O. Box 3640, 76021 Karlsruhe, Germany; \\ ${ }^{\mathrm{e}}$ Karlsruhe Nano Micro Facility, H.-von-Helmholtz-Platz 1, 76344 Egg.-Leopoldshafen, Germany \\ *juliana.solheid@kit.edu; phone +49 721 608-22872
}

\begin{abstract}
Although there have been numerous attempts to define how different laser polishing parameters affect the generated surface roughness, there has been no detailed investigation of how their effects can be combined to optimize the process. This paper applies statistical analysis to model and predict the resulting surface roughness for laser post-processing of components made of Ti-6Al-4V and produced by laser powder bed fusion. This model is based on analysis of a wideranging experimental programme investigating how the interaction of the governing parameters, i.e., laser power, number of repetitions, axial feed rate, scanning speed, and focal position, affected surface roughness. The experimental programme was the result of a robust Design of Experiments analysis and experimental analysis using ANOVA. It is expected that the outcomes will contribute towards the understanding of how the governing parameters influence the laser polishing process and final surface roughness, and would be a tool for optimizing their selection. The results of the ANOVA (analysis of variance) revealed that the most significant parameters are scanning speed followed by laser power and then axial feed rate. In addition, a clear tendency for the estimated Ra to decrease with the increase in laser power at lower values of axial feed rate and of scanning speed, and a focal position in the region of $2 \mathrm{~mm}$. It is noted that the process parameters were varied over wide ranges, including extreme values, which made it difficult to accurately model the dependent variable over the full range of experimental trials.
\end{abstract}

Keywords: laser polishing, surface quality, additive manufacturing, roughness prediction, Ti-6Al-4V

\section{INTRODUCTION}

Additive manufacturing (AM) is a technology in which material is deposited layer upon layer to produce a component or part. Its debut was in 1987 with the emergence of the Stereolithography process. Since then a great variety of AM processes have been introduced, all followed by rapid development from prototyping to production processes. The AM process can reduce material waste and production lead-times, but its key advantage is the possibility of creating complex geometries with internal features, which cannot be produced by traditional processes. These characteristics make the AM process a preferable alternative to conventional processes, especially with its ability for customization and personalization of fabricated components with little impact on cost and complexity of the manufacturing process. Today, the range of materials suitable for use with the AM process is already extensive, and the variety and variations of parts produced by AM is growing steadily with improvement of the AM technologies and development of new materials. AM has proved particularly popular in the biomedical, aerospace and electronics industries [1-3]. Laser-based powder bed fusion (L-PBF) is one AM method for building metallic parts. It consists of repeatedly spreading a thin layer of powdered material over a building platform, which is selectively scanned by a laser source resulting in the quick melting and solidification of the material into the desired shape. The L-PBF process shares most of the advantages of AM but, more importantly, can be used with metallic superalloys that are now commonly utilised in industrial processes because of their outstanding material properties $[4,5]$. 
Despite its many advantages, the L-PBF process is not always able to provide parts that are ready for immediate use. Often the issue is the low surface quality, the high levels of roughness, of the parts produced, which can adversely impact the mechanical properties. The main reasons for such high levels of surface roughness are the powder particles that can attach to the component's surface during the laser process (known as dross), and the fact that the components are built layer-by-layer, each of finite thickness, which can cause a staircase effect in the vertical direction [6]. In most cases, the AM components require several post-processing steps depending on its material composition and its application. It is common for L-PBF built metallic parts to not only require heat treatment for microstructural modifications but also surface treatments such as sandblasting, mechanical polishing, and traditional machining [7,8].

However, laser post-processing, by which a laser beam irradiates a predefined area of a workpiece in order to melt a thin layer, thus smoothing the surface, is a suitable option for the finishing of AM components. Among its most relevant characteristics are high throughput, the absence of mechanical stresses induced during the process due to its contactless nature, the short processing time and the great flexibility, that results from a wide range of systems, parameters and technologies available, all of which make it a viable alternative to mechanical grinding and polishing processes for improving or functionalization of AM parts [9].

By a defined and controlled combination of laser and process parameters, a versatile machining of each type of material becomes possible. The laser process may induce ablation, surface modification by self-organized structuring, and/or remelting of the surface, the latter leading to planarization by material relocation. Macrostructure smoothing can be achieved by applying continuous wave (cw) laser radiation, this process is known as laser polishing (LP) and it is mainly focused on improving the surface quality of AM components via demolishing surface asperities. Although several attempts have been made to examine the effects of laser polishing and the resulting surface roughness, there has been no detailed and thorough investigation of the effect of process parameters on the generated surface, to enable prediction and optimisation of the process responses [10-12].

There are several known means of optimizing process parameters based on experimental data. The "trial and error" approach, although still widely adopted, can be time-consuming and expensive. It is also not a systematic optimization method and the personal experience of the machine operator can play an important role [13]. One way of analyzing the surface modifications of the components during LP is through reliable models. The finite element method (FEM) which develops a detailed numerical model that describes the physical aspects of the procedure, is one modelling approach extensively used to provide a good understanding of the process behavior. One drawback of this method is the complexity of the models and the computational power required to investigate a wide range of process parameters, or different combinations of parameter sets, in a parametric study [14]. Statistical analysis is another approach that optimizes process parameters by developing a regression model that defines the relationship between the input parameters and the output variables. By adopting Design of Experiments (DoE) and statistical analysis, the number of experiments required to describe the process behavior is drastically reduced when compared to the "trial and error" approach, whilst requiring significantly less computational power than FEM. Such methods have been increasingly used for the optimization of various laser-based processes, i.e., laser cladding, welding, and surface hardening [15-17].

In this context, the aim of the current research is to apply a statistical-based method to examine and predict the resulting surface roughness of laser post-processed L-PBF parts made of Ti-6Al-4V. In particular, the approach presented here includes an experimental investigation of the effect of the governing parameters individually, and in combinations, on the generated surface roughness via a series of experimental trials based on a robust DoE analysis and a statistical analysis of the experimental results. The obtained results are used to build a regression model for the prediction of the surface roughness with different combinations of parameters. The outcome of this paper is to help to get a better understanding of the laser polishing process and the influence of the governing parameters on the final surface quality. Furthermore, it will offer an optimization tool for the selection of the appropriate laser polishing process parameters depending on specific predefined requirements of the final roughness values.

\section{MATERIALS AND METHODS}

\subsection{Material}

The material investigated in this work is Ti-6Al-4V, in the form of rectangular blocks, with $55 \times 15 \times 0.5 \mathrm{~mm}^{3}$ of dimension, manufactured via L-PBF (Figure 1(a)). The chemical composition of the material is presented in Table 1. The samples were produces at 3D Systems Leuven, on DMP Flex 350 machine using LaserForm Ti Gr23 (A). 
Table 1. Chemical composition (wt.\%) of the Ti-6Al-4V blocks produced by L-PBF.

\begin{tabular}{|c|c|c|c|c|c|}
\hline $\mathbf{T i}$ & $\mathbf{A l}$ & $\mathbf{V}$ & $\mathbf{C}$ & $\mathbf{N}$ & $\mathbf{O}$ \\
\hline 87.5 & 6.4 & 3.9 & 0.01 & 0.001 & 0.1 \\
\hline
\end{tabular}

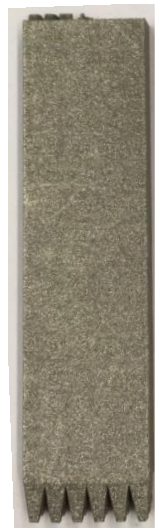

(a)

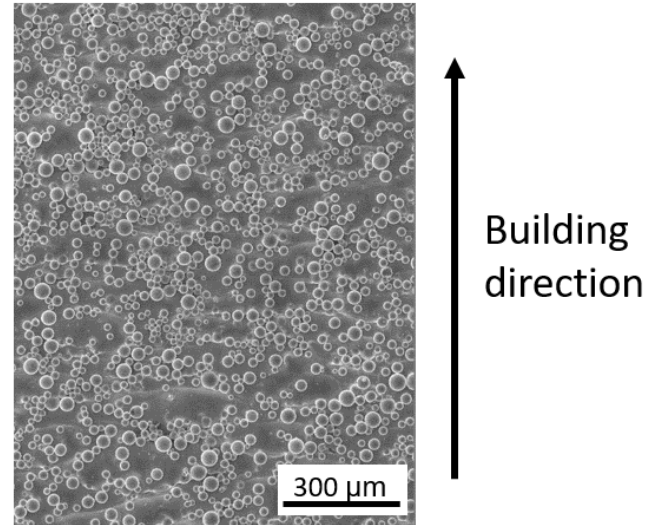

(b)

Figure 1. (a) Picture of the Ti-6Al-4V AM block and (b) SEM image of the side surface of the samples.

\subsection{Laser system and polishing process}

For the laser polishing process, a combination of a TruCell 3010 machine and TruDisk 3001 radiation source (TRUMPF $\mathrm{GmbH}$, laser wavelength $1064 \mathrm{~nm}$ ) was used, which operates in cw mode (Figure 2(a)). The laser beam is focused onto the sample's surface by lenses BEO D70 with focal length of $150 \mathrm{~mm}$. During the process, an argon gas flow was blown on the surface of the sample by a single nozzle in order prevent oxidation of the material's surface.

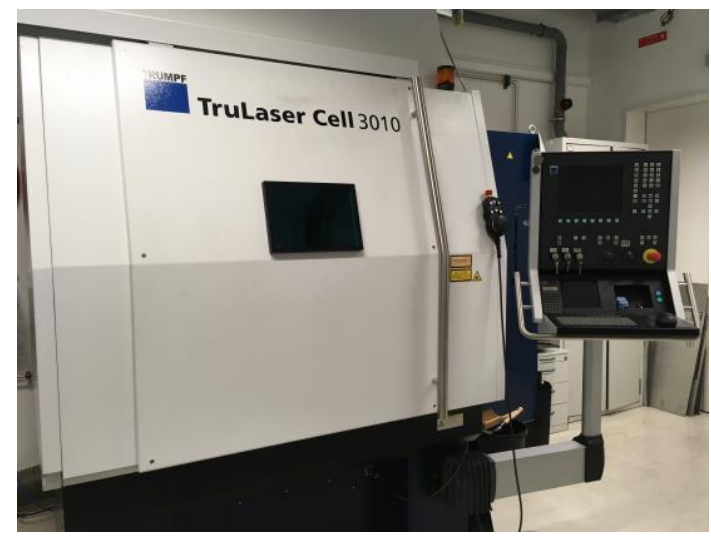

(a)

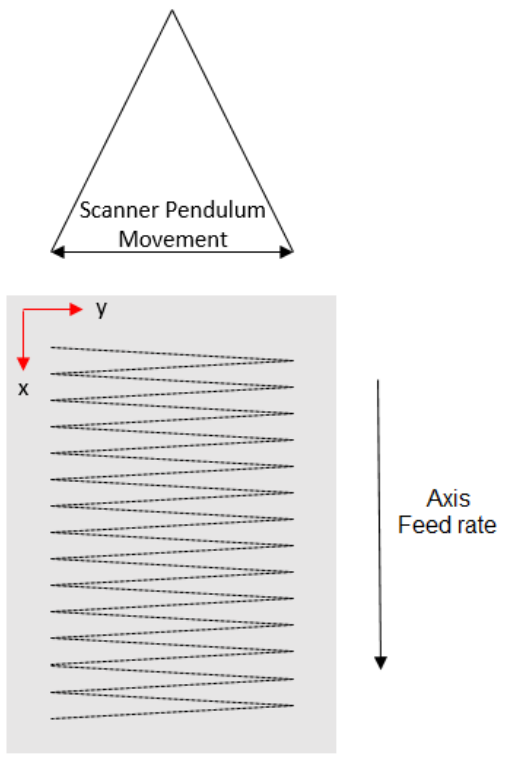

(b)

Figure 2. (a) Image of the laser machine and (b) schematic view of the laser scanning strategy used for laser polishing. 
The laser post-processing was performed on the AM surfaces, which are located vertical to the building direction. This AM surface reveals high degree of roughness due to powder attachment and the layer-by-layer building process, see Figure 1(b). The initial surface roughness (Ra) of the L-PBF samples were in the order of $7 \mu \mathrm{m}$. The LP was executed ex-situ with the parts being removed from the AM machine and processed in a separate laser system.

The governing parameters for the laser polishing process with the given machine are the laser power, the focal position, the number of repetitions (i.e., the total number of times the laser beam traverses the surface), the axial feed rate and the scanning speed. Due to the pendulum movement of the scanning system (Figure 2(b)), the average scanning speed is not directly input in the machine. Instead, this parameter is calculated based on the pendulum frequency and on the adopted amplitude of displacement of the pendulum movement in the y-direction, as shown in Figure 2(b).

\subsection{Design of Experiments}

By using DoE techniques, it is possible to define individual and interactive effects of several factors that influence the experimental results. One advantage of adopting DoE is the possibility of identifying a set of experiments that will provide maximum information. Here, the five parameters listed in the previous section were selected as the five factors of an orthogonal array (OA) design for the experimental trials, see Table 2. A feature of OA is that each factor pair exists an equal number of times, creating a balanced repetition of levels. In this study an L125 OA design was followed that resulted in 125 different sets of parameters for the 125 experimental trials. The range of variation (levels) for each parameter is shown in Table 2, along with their respective normalized values.

Table 2. DoE parameter values selected for the laser polishing tests.

\begin{tabular}{|c|c|c|c|c|c|}
\hline Value & $\begin{array}{c}\text { Laser } \\
\text { Power } \\
(\mathbf{W})\end{array}$ & $\begin{array}{c}\text { Focal } \\
\text { Position } \\
(\mathbf{m m})\end{array}$ & $\begin{array}{c}\text { No. } \\
\text { Repetitions }\end{array}$ & $\begin{array}{c}\text { Axial } \\
\text { Feed } \\
\text { rate } \\
(\mathbf{m} / \mathbf{m i n})\end{array}$ & $\begin{array}{c}\text { Scanning } \\
\text { Speed } \\
(\mathbf{m m} / \mathbf{s})\end{array}$ \\
\hline-1 & 100 & 0 & 1 & 0.3 & 400 \\
\hline-0.5 & 200 & 1 & 2 & 0.45 & 800 \\
\hline 0 & 300 & 2 & 3 & 0.6 & 1200 \\
\hline 0.5 & 400 & 3 & 4 & 0.75 & 1600 \\
\hline 1 & 500 & 4 & 5 & 0.9 & 2000 \\
\hline
\end{tabular}

\subsection{Statistical analysis}

The data processing and statistical modelling were performed with MATLAB (R2018b, MathWorks). A quadratic regression method was used to fit a second order polynomial, Equation (1), to the experimental data.

$$
y=b_{0}+\sum b_{i} x_{i}+\sum b_{i i} x_{i i}^{2}+\sum b_{i j} x_{i} x_{j}
$$

Where ' $y$ ' corresponds to the objective function, or output variable, 'b0','bi','bii' and 'bij' are the regression coefficients, or predictors, ' $n$ ' is the number of factors and ' $x i$ ' is the value of the $i^{\text {th }}$ factor. Regression coefficients were estimated using the least-square method. For determination of the significance of the coefficients and the suitability of the proposed model, analysis of variance (ANOVA) was adopted using a confidence level of $95 \%$.

\subsection{Surface metrology system}

The surface measurements were performed and filtered in accordance with the EN ISO 4288 standard for 2D roughness profile measurements which requires a stylus travel of $15 \mathrm{~mm} ; 12.5 \mathrm{~mm}$ evaluation length, $2.5 \mathrm{~mm}$ of sampling length, with a cut-off of $2.5 \mathrm{~mm}$. The equipment used was a combination of a MarSurf XR 1 tactile surface measuring unit and its drive unit MarSurf GD 26 with the probe arm BFW A 10-45-2/90 ${ }^{\circ}$. The parameter chosen as the quality mark to be 
used in the analysis performed in this work was the arithmetic mean height (Ra), which indicates the average of the absolute value along the sampling length.

\section{RESULTS AND DISCUSSION}

\subsection{Model coefficient estimation}

The first step in the statistical analysis is the estimation of the coefficients in quadratic regression model, Equation (1). That required the adoption of a robust regression method, which is less affected by outliers. The chosen fitting method works by assigning weight to the data points (see Figure 3) iteratively through a reweighted least squares method. The result is a model that is less sensitive to large changes in small parts of the data than obtained via ordinary least squares.

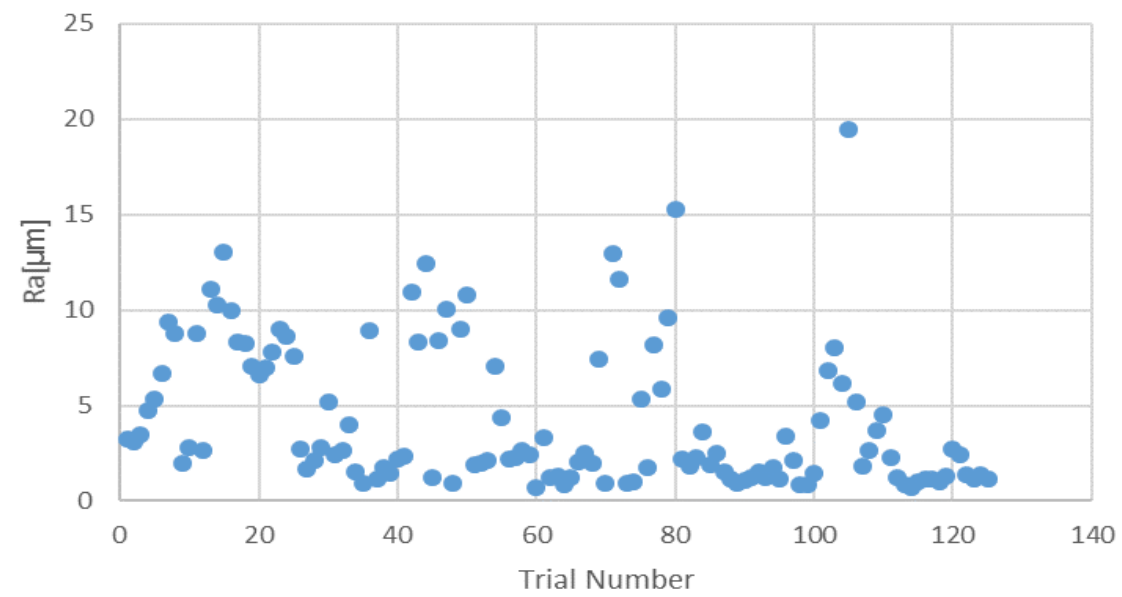

Figure 3. Ra results for each experimental trial.

The robust fitting starts by assigning equal weight to each point, the model coefficients are then estimated using the ordinary least squares method. The following iterations weight each point so that the ones farther from the model predictions in the preceding iteration are given lower weight. The process continues until the estimated values of the coefficients converge within a specified tolerance.

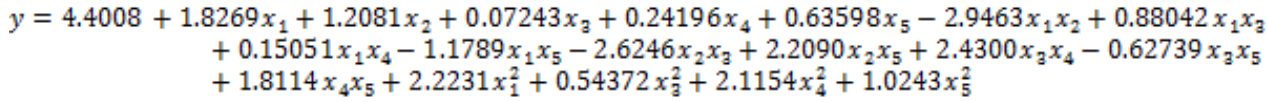

The model resulting from the quadratic linear regression model with a robust fit presents 21 terms for the 5 predictors listed in Section 2.2. The values obtained for each coefficient are presented in Equation (2), where ' $\mathrm{x}_{1}$ ' is the laser power, ' $\mathrm{x}_{2}$ ' is the focal position, ' $\mathrm{x}_{3}$ ' is the number of repetitions, ' $\mathrm{x}_{4}$ ' is the axial feed rate, and ' $\mathrm{x}_{5}$ ' is the scanning speed. This equation is further used to describe the interaction between different process parameters and to predict the Ra roughness of AM components submitted for laser polishing with different set of parameters.

\subsection{Parameters interaction}

As mentioned in the previous section, the model described was used to generate interaction plots as shown in Figure 4. From the total of 20 possible interaction plots, 12 were selected to be presented here based on the ANOVA results (in section 3.4) of the most significant parameters. The plots show the estimated behavior of the interaction between two specified variables, and were obtained by varying the one parameter at different levels of the other variable. From the output values of the dependent variable in each of these graphs, it is possible to observe how sensitive it is the interaction between two different variables.

Figure 4 shows the variation of the estimated Ra with specific pairs of parameters. Each pair of parameters is represented in two interaction graphs. For example, the top two panels are for Laser Power and Scanning Speed. In the panel on the upper left, the power was held constant at $100 \mathrm{~W}$, then $300 \mathrm{~W}$ and finally $500 \mathrm{~W}$, and for each of these power values the scanning speed was varied from 400 to $2000 \mathrm{~mm} / \mathrm{s}$. In the panel on the upper right the Scanning Speed was held at 400 
$\mathrm{mm} / \mathrm{s}$, then $1200 \mathrm{~mm} / \mathrm{s}$ and then $4000 \mathrm{~mm} / \mathrm{s}$, while for each scanning speed the laser power varied from $100 \mathrm{~W}$ to $500 \mathrm{~W}$. Similarly for the combinations of Feed rate - Scanning Speed, Scanning Speed - Feed Rate, Power - Feed Rate, Feed Rate - Power, Speed - Focal position, Focal position - Speed, Focal position - Feed rate, Feed rate - Focal position, Power - Focal position, and Focal position - Power.
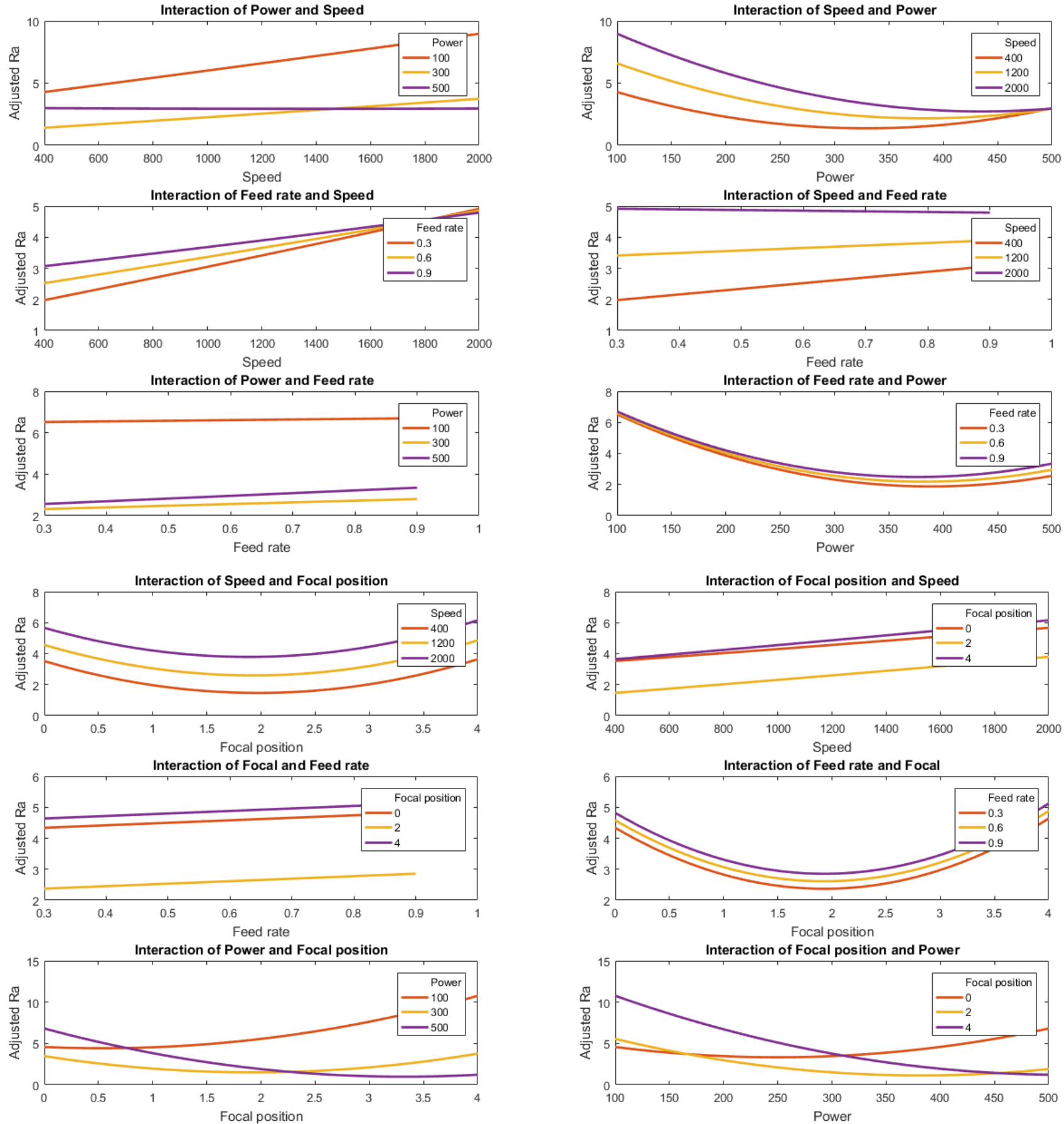

Figure 4. Interaction plots of the most significant laser polishing parameters.

It can be seen from Figure 4 that, there is a clear tendency for the estimated Ra to decrease: with higher values of laser power; with lower values of axial feed rate and of scanning speed; and a focal position in the region of $2 \mathrm{~mm}$. However, these are general trends and have to be considered in their specific circumstances. For example, while it is true that, 
generally, a focal position of about $2 \mathrm{~mm}$ gives minimal Ra, we see that at the highest laser power (500 W) minimum Ra occurs at a focal position of $3.5 \mathrm{~mm}$, and at least laser power $(100 \mathrm{~W})$ minimum Ra occurs at a focal position close to zero. The particular combination for minimum Ra must be selected carefully.

\subsection{Roughness prediction}

Figure 5 shows a prediction slice plot obtained from the developed model. Each of the five curves shown represents the change in the dependent variable as a function of the value of the input parameter. The dashed boundaries are the $95 \%$ confidence limits for the predicted value of the dependent variable. According to the statistical model adopted, it is possible to obtain a laser polished surface with a Ra value of around $0.5 \mu \mathrm{m}$. The suggested set of parameters to achieve this result is as in Figure 5, the corresponding normalized values for each process parameter are: laser power $=0.5$ $(400 \mathrm{~W})$, focal position $=0.5(+3 \mathrm{~mm})$, number of repetitions $=0.8(5$ repetitions $)$, axial feed rate $=-0.5(0.45 \mathrm{~m} / \mathrm{min})$ and scanning speed $=-1(400 \mathrm{~mm} / \mathrm{s})$. Considering that the initial Ra values of the Ti-6Al-4V samples used in this work were in the range of $7 \mu \mathrm{m}$, the prescribed set of parameters would represent a significant improvement.

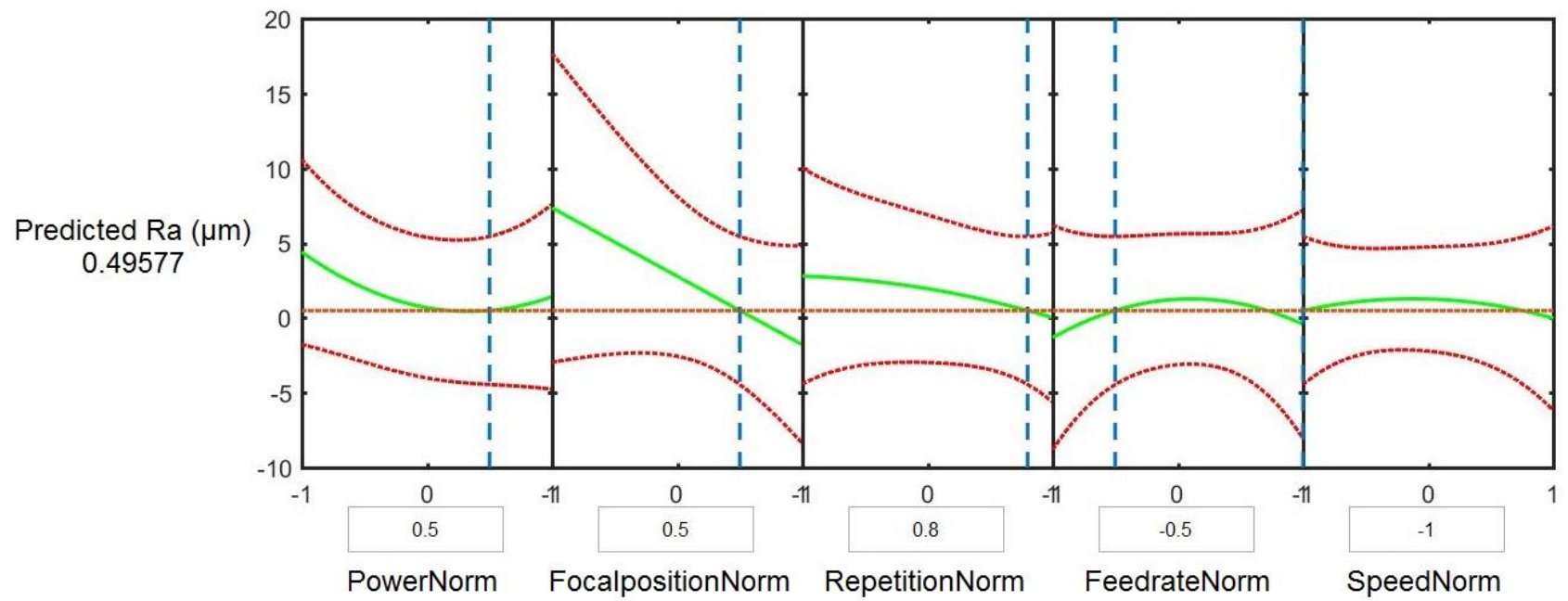

Figure 5. Prediction slice plot with the normalized values for each parameter.

Besides the set of laser polishing parameters cited above, other combinations could provide a reduction in surface roughness. Different applications can require different values of surface roughness, and prediction slice plots can be used either to minimize or to select the most suitable value of the surface roughness, even if not the minimum. Being able to select the laser polishing process parameters to obtain a smoother surface, in the desired roughness range, would reduce the post-processing of AM components.

\subsection{Analysis of variance (ANOVA)}

The suitability of proposed model was analyzed using ANOVA. Table 3 presents the values obtained from the sets of experimental data points. The Error DoF (error degrees of freedom) is the number of coefficients subtracted from the number of observations, the RMS error (root mean squared error) estimates the standard deviation of the error distribution, the R-squared and adjusted R-squared are the coefficient of determination and adjusted coefficient of determination, respectively, and suggests percentage of variability in the response that is explained by the model and, finally, the p-value is the value of significance of the F-test for the model.

The present model was based on 125 experimental observations and, as mentioned in Section 3.1, the equation presented 21 terms, resulting in 104 error degrees of freedom. However, as the value of two of the coefficients is zero, see Table 3 , the equation for the error degrees of freedom estimation is considered to have only 19 terms. The standard deviation of the error is 2.35 , while the model explains $61.7 \%$ and $55.2 \%$ of the variability of the response and adjusted response, respectively. Finally, the value of 5.65e-15 (almost zero) for the p-value indicates that the model is so significant at the $5 \%$ significance level. From the p-value analysis of each parameter individually, presented in Table 4 the most significant parameters are the scanning speed, laser power and feed rate, while the focal position and the number of repetitions are not that significant. 
Table 3. ANOVA analysis values for the adopted statistical model.

\begin{tabular}{|c|c|c|c|c|c|}
\hline $\begin{array}{c}\text { No. of } \\
\text { observations }\end{array}$ & $\begin{array}{c}\text { Error } \\
\text { DoF }\end{array}$ & $\begin{array}{c}\text { RMS } \\
\text { error }\end{array}$ & $\mathbf{R}^{\mathbf{2}}$ & $\begin{array}{c}\text { Adjusted } \\
\mathbf{R}^{2}\end{array}$ & p-value \\
\hline 125 & 106 & 2.35 & 0.617 & 0.552 & $5.65 \mathrm{e}-15$ \\
\hline
\end{tabular}

Table 4. p-value analysis for the laser polishing parameters.

\begin{tabular}{|c|c|}
\hline Parameter & p-value \\
\hline Speed & $8.5966 \mathrm{e}-09$ \\
\hline Power & $2.3425 \mathrm{e}-08$ \\
\hline Feed rate & 0.018873 \\
\hline $\begin{array}{c}\text { Focal } \\
\text { position }\end{array}$ & 0.10462 \\
\hline Repetition & 0.70039 \\
\hline
\end{tabular}

\section{CONCLUSIONS}

This paper has reported the development of a statistical model to enhance the understanding of, and predict the effects on, surface roughness of the surfaces of AM components subject to laser polishing. The model considered the effect and interactions of laser power, focal position, axial feed rate, scanning speed, and number of repetitions, and how these influenced the predicted surface roughness, Ra, of the resulting component. It was found that the most significant parameters, amongst the ones analyzed, are the scanning speed, laser power and axis feed rate. Also, there was a clear tendency for Ra to decrease with higher values of laser power under lower values of feed rate and of speed, and a focal position in the region of $2 \mathrm{~mm}$. However, the process parameters were varied over wide ranges, including extreme values which made it difficult to produce a model that accurately modelled/simulated the dependent variable over the full range of experimental results. Nevertheless, the authors are convinced that the results produced will be of assistance to those working with AM and concerned with obtaining minimum surface roughness by adjusting the value of the process parameters. Future work could investigate improving statistical model by, e.g., implementing different regression methods. Developing more reliable, validated models would enable laser polishing to become a process based on informed decision making.

\section{ACKNOWLEDGMENTS}

This work has received funding from the European Union's programme PAM ${ }^{2}$ within Horizon 2020 under grant agreement No. 721383. Furthermore, the support for laser materials processing by the Karlsruhe Nano Micro Facility (KNMF, http://www.knmf.kit.edu/), a Helmholtz research infrastructure at the Karlsruhe Institute of Technology (KIT), is gratefully acknowledged.

\section{REFERENCES}

[1] Yang, L., Hsu, K., Baughman, B., Godfrey, D., Medina, F., Menon, M., and Wiener, S., Additive manufacturing of metals: the technology, materials, design and production, Springer Series in Advanced Manufacturing (2017). 
[2] Chen, L., He, Y., Yang, Y., Niu, S., and Ren, H., "The research status and development trend of additive manufacturing technology." Int. J. Advanced Manufacturing Technologies, 89, 3651-3660 (2017).

[3] Guo, N., and Leu, M., "Additive manufacturing: technology, applications and research need." Frontiers of Mechanical Engineering, 8(3), 215-243 (2013).

[4] Herzog, D., Seyda, V., Wycisk, E., and Emmelmann, C., "Additive manufacturing of metals.” Acta Materialia, 117, 371-392 (2016).

[5] Bourell, D., Kruth, J.P., Leu, M., Levy, G., Rosen, D., Beese, A.M., and Clare A., "Materials for additive manufacturing." CIRP Annals - Manufacturing Technology, 66, 659-681 (2017).

[6] Charles, A., Elkaseer, A., Thijs, L., Hagenmeyer, V., and Scholz, S., "Effect of Process Parameters on the Generated Surface Roughness of Down-Facing Surfaces in Selective Laser Melting.” Applied Sciences, 9(6), 1256 (2019).

[7] Townsend, A., Senin, N., Blunt, L., Leach, R.K., and Taylor, J.S. "Surface texture metrology for metal additive manufacturing: a review." Precision Engineering, 46, 34-47 (2016).

[8] Haefele, T., Schneberger, J.H., Kaspar, J., Vielhaber, M., and Griebsch, J., "Hybrid Additive Manufacturing Process Chain Correlations and Impacts.” Procedia CIRP, 84, 328-334 (2019).

[9] Elkaseer A., Lambarri J., Quintana I., and Scholz S., "Laser Ablation of Cobalt-Bound Tungsten Carbide and Aluminium Oxide Ceramic: Experimental Investigation with ANN Modelling and GA Optimisation." Smart Innovation, Systems and Technologies 130, 21-30 (2018).

[10]Ma, C.P., Guan, Y.C., and Zhou, W., "Laser polishing of additive manufactured Ti alloys.” Optics and Laser in Engineering, 93, 171-177 (2017).

[11]Bhaduri, D., Penchev, P., Batal, A., Dimov, S., Soo, S.L., Sten, S., Harrysson, U., Zhang, Z., and Dong, H., "Laser polishing of 3D printed mesoscale components." Applied Surface Science, 405, 29-46 (2017).

[12] Marimuthu, S., Triantaphyllou, A., Antar, M., Wimpenny, D., Morton, H., and Beard, M., "Laser polishing of selective laser melted components.” Int. J. Machine Tools and Manufacture, 95, 97-104 (2015).

[13] Muhammad, K.F., and Yusoff, W.A.W., "Optimization of laser cutting parameters using variable weight Grey-Taguchi method.” Australian Journal of Basic and Applied Sciences, 8 (15), 361-365 (2014).

[14] Bayat, M., Mohanty, S., Hattel, J.H., "Multiphysics modelling of lack-of-fusion voids formation and evolution in IN718 made by multi-track/multi-layer L-PBF.” Int. J. Heat and Mass Transfer, 139, 95-114 (2019).

[15] Sun, Y., and Hao, M., "Statistical analysis and optimization of the process parameters in Ti6Al4V laser cladding using Nd:YAG laser.” Optics and Lasers in Engineering, 50, 985-995 (2012).

[16] Benyounis, K.Y., and Olabi, A.G., "Optimization of different welding processes using statistical and numerical approaches - A reference guide.” Advances in Engineering Software, 39, 483-496 (2008).

[17] Hamad, A.R., Abboud, J.H., Shuaeib, F.M., and Benyounis K.Y., "Surface hardening of commercially pure titanium by laser nitriding: Response surface analysis.” Advances in Engineering Software, 41(4), 674-679 (2010). 